\title{
Liderazgo femenino en los diarios digitales $E I$ Comercio y El Universo de Ecuador
}

\section{Female leadership in the Ecuadorian online newspapers El Comercio and El Universo}

\author{
Baquerizo Neira, G. y Umpierrez de Reguero, S. ${ }^{1}$ \\ Recibido: 30-09-2018 - Aceptado: 25-01-2019 \\ DOI: https://doi.org/10.26441/RC18.1-2019-A2
}

\begin{abstract}
RESUMEN: En los últimos años, la presencia femenina ha aumentado en las instituciones políticas ecuatorianas. Sin embargo, la visibilidad de la mujer en el espacio público continúa siendo limitada. En este contexto, resulta pertinente investigar cómo estas mujeres son representadas en los medios digitales. Para ello, se realiza un estudio descriptivo de la caracterización socio-demográfica de las mujeres en la opinión publicada en los diarios digitales El Universo y El Comercio. Se identifica en qué medida estos medios se refieren a las mujeres como fuentes de noticia. De un número de 2.203 notas de prensa publicadas entre 2010 y 2015, se identifica la frecuencia de aparición de 78 mujeres consultadas como fuentes periodísticas. Entre los resultados se observa que las mujeres con más apariciones en los medios digitales en gran medida oscilan entre los 35 y 50 años de edad y poseen estudios universitarios. Más de la mitad son mestizas.
\end{abstract}

Palabras clave: mujeres; medios digitales; Ecuador; perfil socio-demográfico; comunicación política; descarga de datos.

\begin{abstract}
Last couple of years, female presence has increased in Ecuadorian political institutions. However, the visibility of women in the public media continues to be limited. In this context, we investigate how these women are represented by the digital media. We conducted a descriptive study of the socio-demographic characterization of women in published opinion in the digital newspapers El Universo and El Comercio. We identify to what extent these media take women as a reference in their publications, and what are the female profiles in this regard. Through a number of 2,203 pressreleases published between 2010 and 2015, we analyze the frequency of appearance of 78 women referred as sources of news. In general, we observe that women consulted as journalistic sources in digital newspapers fluctuate between 35 and 50 years of age, more than $50 \%$ identify themselves as mestizas, and most of them have university studies.
\end{abstract}

Key words: women; digital media; Ecuador; socio-demographic profile; political communication; data crawling.

1 Gabriela Baquerizo Neira es Doctora en Comunicación Social por la Universidad Austral y Profesora Asociada en el área de Comunicación Estratégica y Relaciones Públicas en la Facultad de Comunicación de la Universidad Casa Grande (Ecuador). gbaquerizo@casagrande.edu.ec, https://orcid.org/0000-0002-4837-1076

Sebastián Umpierrez de Reguero es Magíster en Ciencia Política, con mención en Relaciones Internacionales de la Pontificia Universidad Católica de Chile y doctorando en la Facultad de Ciencias Sociales e Historia de la Universidad Diego Portales (Chile). sebastian.umpierrez@mail.udp.cl, https://orcid.org/0000-0002-5464-0690 


\section{Introducción}

Aunque en Ecuador ha habido un avance sustancial en materia de derechos para la participación y representación femenina en la vida pública y política (Castellanos y Ulloa, 2016; Umpierrez de Reguero, Jara-Alba y Cassis Jurado, 2016), la presencia efectiva de la mujer en los medios de comunicación tradicionales y no tradicionales es aún limitada y persisten los estereotipos de género. En efecto, se coincide con la afirmación de Barba y Nieto (2006) cuando refieren que el liderazgo femenino se ha caracterizado por una marcada desigualdad frente a las condiciones masculinas en todos los ámbitos de la sociedad. De ahí que, por ejemplo, en el ranking de las 100 entidades extraídas de los medios digitales con mayor cobertura (El Universo y El Comercio), solo el $14 \%$ sean mujeres y ni siquiera se ubique una de ellas, entre las diez primeras posiciones ${ }^{2}$.

En este artículo se caracteriza el perfil sociodemográfico de las mujeres más representadas por la opinión publicada ecuatoriana como fuentes de noticia, en el periodo 2010 - 2015. Esta identificación se realizó por medio de la extracción de 2.203 notas de prensa (crawling de datos) de El Universo y El Comercio, en donde se registraron $78(17,6 \%) \mathrm{mu}-$ jeres y $366(82,4 \%)$ hombres de un total de 444 personas.
Esta investigación pretende contribuir a las discusiones abiertas sobre la presencia y participación efectiva de la mujer en los medios de comunicación digitales latinoamericanos, a través del estudio de caso ecuatoriano. Por una parte, se espera visibilizar el rol de las mujeres en los medios digitales de nuevas democracias, $y$, por otra parte, se aporta al acervo bibliográfico en materia de comunicación digital, opinión publicada y género.

Adicional a esta introducción, se busca revisar brevemente la participación de la mujer en el ámbito mediático y de opinión publicada. Luego, se declara la metodología a seguir, en donde no sólo se explica el proceso de crawling $^{3}$ de datos, sino también se identifican las variables de interés para la caracterización sociodemográfica de la mujer en medios digitales ecuatorianos de mayor cobertura.

\section{Visibilizando a las mujeres en los medios digitales}

En Ecuador, los resultados oficiales de los últimos comicios presidenciales celebrados en febrero de 2017 evidenciaron un porcentaje significativo de mujeres en el ámbito legislativo. Por tercera vez en la historia política ecuatoriana, la representación femenina en la Asamblea Nacional fluctuó entre el $35 \%$ al $40 \%$ del total de curules (Consejo

2 Esta afirmación responde a los datos extraídos de los diarios digitales El Comercio y El Universo entre los años 2010-2015.

3 Una araña web (crawler) es un programa o script automatizado que inspecciona la World Wide Web de una manera metódica y automatizada. Las arañas web son utilizadas principalmente para crear una copia de todas las páginas visitadas para su procesamiento posterior con un motor de búsqueda que creará un índice de las páginas descargadas para proveer búsquedas más rápidas. 
Nacional Electoral, 2017). Sin embargo, en Ecuador todavía existen claras desventajas de las mujeres frente a los hombres en temas como: la equidad en la remuneración, los horarios de trabajo, las actividades que desempeñan, entre otros factores (Gender Gap Report, 2016; El Comercio, 9 de marzo de 2018). Con estos antecedentes y tomando en consideración que la población femenina ecuatoriana constituye el 50,4\% (INEC, 2010), resulta interesante caracterizar a las mujeres representadas por la opinión publicada; es decir, si los medios están tomando como referencia sus opiniones, y qué perfil proyectan de ellas.

A nivel latinoamericano, el acceso que tienen las mujeres a los medios de comunicación para transmitir sus opiniones políticas a la sociedad es mínimo (Llanos, 2014). La presencia masculina en relación a la femenina en los medios continúa siendo mayoritaria. La aparición de las mujeres como sujeto de la noticia no supera el 30\% de los casos, tanto en medios tradicionales (es decir, radio, televisión o prensa escrita) como en plataformas digitales de comunicación y (plataformas de) redes sociales (GMMP, 2015).

De acuerdo con el reporte del Proyecto Global de Monitoreo de Medios (GMMP), en el que participaron catorce países de Latinoamérica y entre los que se encontraba Ecuador, la cobertura mediática de temas relacionados con el sexo femenino se hace en forma estereotipada y se lo relaciona con temáticas como: salud, natalidad, belleza, entretenimiento, entre otras; mientras que, a los hombres se los asocia con noticias sobre economía y política. Se evidencia que, a pesar de que los medios en sus noticias abordan temas como el trabajo de la mujer en el campo o su participación en la vida política, las voces expertas e imágenes que a menudo se muestran para graficar la noticia, son masculinas (GMMP, 2015).

En América Latina, pese a que la participación de las mujeres en la arena política ha ido en aumento durante la última década. Inclusive, ellas se han logrado ubicar en altos cargos de gobierno como presidentas, ministras, embajadoras, entre otros (por ejemplo, Michelle Bachelet, Cristina Fernández de Kirchner, Dilma Rousseff), su representación en los medios continúa marcada por rasgos de inequidad. Así, cuando los medios las refieren como sujetos de la noticia o fuentes de información, aún hacen énfasis en su estado civil e incluso en su maternidad, lo que en los casos de las líderes políticas en ocasiones puede "afectar la postura de sus electores" (Bystrom, 2004: 19).

De igual forma, la presencia femenina en los medios, tanto en las publicaciones, como en el ejercicio profesional como reporteras, es menor en relación con la masculina (GMMP, 2015). Incluso, en ocasiones, su participación en medios está supeditada por la edad. El GMMP (2015) al identificar los grupos etarios de mujeres en Ecuador, determinó que la brecha de género se mantiene.

Como voceras, el $10 \%$ de mujeres y $36 \%$ de hombres se encuentran en una edad comprendida entre 19 a 34 años, similar situación se observa en el 
rango de 35 a 49 años donde las mujeres son el $14 \%$ y los hombres el $51 \%$. Como expertas o comentaristas el $10 \%$ de mujeres y el $7 \%$ de hombres se encuentran entre los 19 y 34 años, entre los 35 y 49 años se igualan en porcentaje mujeres y hombres con el $14 \%$ pero en una edad de 50 a 64 años las mujeres no tienen representación (0\%) mientras los hombres aún constituyen el $10 \%$. Generalmente en estudio de televisión, la edad de reporteros y presentadores, oscila entre los siguientes rangos: 19-34 años (6) mujeres 18\% hombres 0\%; 35-49 años $71 \%$ son mujeres (24) y $85 \%$ hombres (29); de 50-64 años mujeres 12\% y de 65 años o más 15\% solo de hombres (GMMP, 2015: s/n).

La brecha que existe entre los dos géneros como conceptualización binaria (femenino y masculino), es evidente frente a su participación en los medios y la relevancia que tiene la variable edad-en el caso de la práctica periodística femenina en medios audiovisuales-como elemento fundamental para asumir el rol de anchor de televisión (Ross y Carter, 2011).

Otro elemento a considerar en la inequitativa participación femenina en los medios es la cualificación, siendo que, para los periodistas "la selección de la fuente es una de las principales decisiones del encuadre de la noticia” (Niemi y Pitkänen, 2017: 1). Aquí cabe preguntarse: ¿Cuál es el proceso de selección de la fuente de una noticia? - ¿Cómo los medios determinan la credibilidad $y$ competencia que tiene un individuo para emitir comentarios sobre un tema?
Expertos sostienen que esta selección en ocasiones puede estar determinada por el inexistente reconocimiento de "mujeres en puestos expertos en la sociedad (y, especialmente, en el mundo académico)"; así, la selección de fuentes referentes sobre una temática es producto de estereotipos masculinos (Niemi y Pitkänen, 2017: 2).

\section{Metodología}

¿El perfil sociodemográfico puede llegar a incidir en la visibilidad de la mujer en la opinión publicada por los medios digitales? Para aproximarse a responder esta pregunta, se realizó inicialmente una selección de notas de prensa para identificar la frecuencia y porcentaje de aparición de mujeres en los medios digitales seleccionados para el estudio. Por una parte, el diario $E l$ Comercio posee una versión impresa y tiene un amplio tiraje nacional. Este medio en su versión digital está ubicado en el tercer lugar del ranking de tráfico web Alexa 2019 del top sites de Ecuador, y en el primer puesto en el mismo índice, si solo se considera a los medios de comunicación nacional. Por otra parte, el diario El Universo, periódico guayaquileño, que también posee un tiraje impreso significativo, si no a nivel nacional, con gran presencia más allá de Guayaquil, está ubicado en el séptimo lugar del ranking Alexa 2019. Este diario digital figura en segunda posición en el ranking Alexa, si solo se considera a los medios de comunicación nacional.

La selección de las notas de prensa se formuló mediante la implementación de un algoritmo de búsqueda con el que se 
extrajo de forma automática de los diarios mencionados las 2.203 notas de prensa para la posterior identificación de entidades. En el caso de El Universo, se seleccionaron las secciones: Noticias, Gran Guayaquil, Ecuador, Economía e Internacional; así se capturó el ámbito local como noticias de entretenimiento y asuntos coyunturales de interés nacional e internacional. Esto se realizó a partir de un patrón de registro hacia todas las URLs (Uniform Resource Locator) disponibles del sitio web: https://www.eluniverso.com. Gracias a que la página del diario presenta un servicio de archivos que habilita el acceso a las noticias del día a través de la fecha de publicación, este medio en versión digital permitió generar el patrón de búsqueda, que se hizo con el siguiente formato: http://www. eluniverso.com/servicios/archivo/AÑ/ MES/DIA.

En el caso de El Comercio, la extracción también se identificó mediante un patrón de búsqueda hacia todas las URLS disponibles del sitio web: http:// www.elcomercio.com. Ahora bien, como este medio posee una búsqueda paginada de artículos, fue conveniente incorporar un filtro por el intervalo de las fechas deseadas de las secciones escogidas para el estudio-Opinión y Actualidad-por considerarse una selección diversa de contenidos internacionales, editoriales y nacionales. Una vez hecha la extracción, también se organizaron los datos de la misma manera que en El Universo.

Una vez encontrado el patrón de búsqueda de cada uno de los diarios, se realizó el crawling y se recopilaron todas las noticias que se publicaron entre enero de 2010 y diciembre de 2015, en ambos diarios. Después de la extracción de datos, cada procesamiento se archivó jerárquicamente por fechas y luego por tópico en un archivo.txt con el siguiente formato: título, tópico, etiquetas (tags), y cuerpo de la noticia (ver Figura 1).

Después de la extracción de las notas de prensa, se empleó la herramienta Stanford NER, para detectar y clasificar los nombres de las personas. De esta forma, por una parte, se pudo identificar a las mujeres y, por otra parte, se cuantificó su frecuencia de aparición mediática. Así, este proceso permitió identificar que: de un total de 444 personas, solamente 78 $(17,6 \%)$ eran mujeres (ver Anexos 1), y que los medios las referían como expertas en las notas de prensa publicadas por ambos diarios digitales.

La Tabla 1 indica que la media de la frecuencia de aparición es de 255 referencias, de forma agregada en $E l$ Universo y en El Comercio. No obstante, la dispersión de respuesta es relevante según se puede observar en el resultado de la desviación estándar. En este sentido, las respuestas oscilan entre 105 y 986 apariciones. En efecto, la entidad que ocupó el valor máximo de la muestra es Lourdes Tibán (ex Asambleísta Nacional y lideresa indígena); en cambio, Ruth Seni (Presidenta de la Corte Constitucional) y Jeannette Sánchez (ex Ministra de Bienestar Social y Ministra Coordinadora de Política Económica), se posicionaron en las últimas posiciones del ranking total extraído del crawling de datos (es decir, 443 y 444 , respectivamente). 
Figura 1. Crawling de datos diarios digitales e identificación de entidades
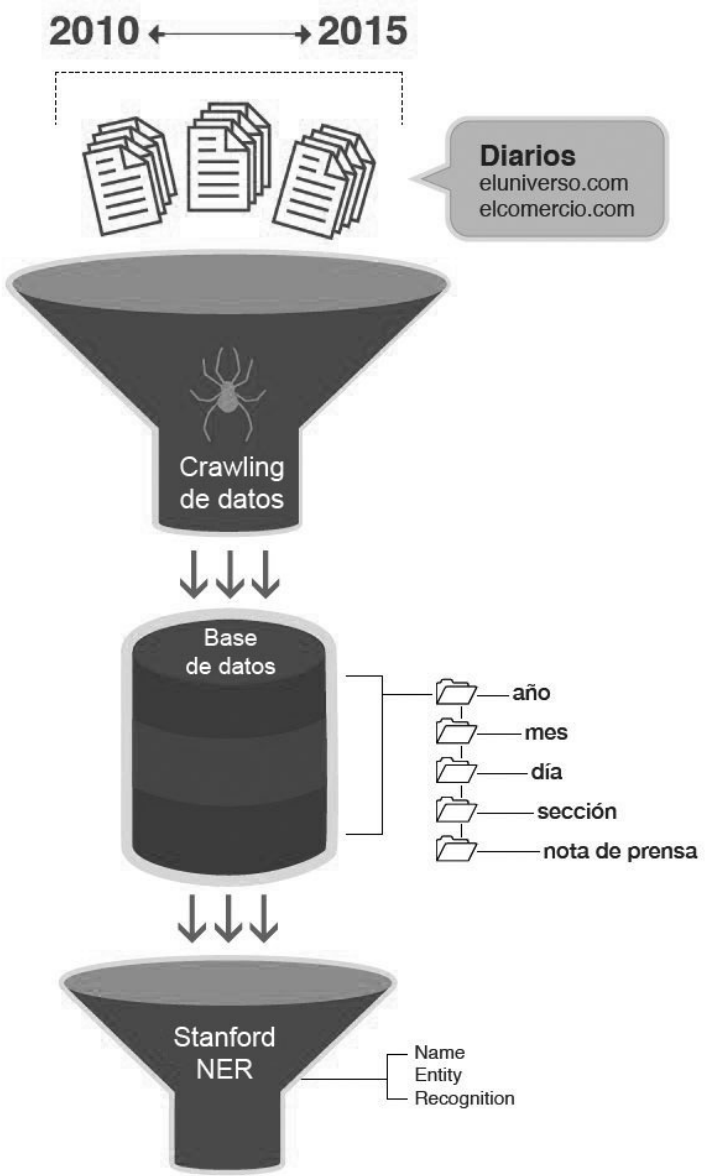

$\downarrow \downarrow \downarrow$

Identificación de entidades por frecuencia de aparición en diarios

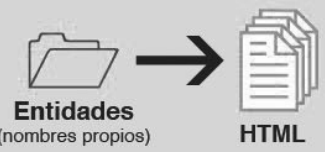


Tabla 1. Estadísticos descriptivos de la variable dependiente

\begin{tabular}{|l|c|c|c|c|}
\hline & Media & Desviación Estándar & Valor mínimo & Valor máximo \\
\hline Frecuencia de aparición & 254,58 & 171,31 & 105 & 986 \\
\hline
\end{tabular}

Fuente: Elaboración propia

\section{Resultados}

El perfil sociodemográfico de las mujeres en la opinión publicada, a partir del análisis de las notas de prensa de $E l$ Universo y El Comercio (versión digital) responde a la categoría teórica de reputación denominada "capital humano". Esta "argumenta que las personas generan valor mediante la adquisición de conocimientos, habilidades y credenciales a través de logros educativos y experimentales (Zinko et al., 2007: 176). Asimismo, esta categoría supone que el prestigio de las instituciones y empresas donde se formaron y trabajaron se traslada a la persona. En efecto, la identificación del perfil socio-demográfico de estas mujeres permite caracterizar la figura femenina que seleccionan los diarios digitales como fuente de información en temas políticos, económicos y sociales.

Se pudo constatar que en la agenda mediática ecuatoriana hay una mayor inclusión de hombres que de mujeres como fuentes periodísticas en los diarios digitales. Esto concuerda con García Beaudoux (2017) que sostiene que "el $71 \%$ de los expertos consultados como fuentes periodísticas o invitados a opinar en los medios son hombres, a pesar de que en muchos países las mujeres conforman la mayoría de la población de los graduados universita- rios" (p. 61). Con ello, se entrevé que en Ecuador al igual que en América Latina, aún existe una brecha de género entre hombres y mujeres en la participación pública, y que prevalece la opinión de la figura masculina sobre la femenina (Barba y Nieto, 2006).

Bajo este contexto y una vez identificado el número de observaciones $(\mathrm{N}=$ 78 mujeres), se levantó una base de datos con las siguientes variables: nombre completo, edad, lugar de nacimiento, nacionalidad, raza, educación, título, estado civil y número de hijos. A partir del análisis de estas variables se exploraron las siguientes interrogantes: ¿Qué cualificación tienen las mujeres referidas en la opinión publicada? ¿Cuál es la auto-identificación étnica que predomina en las mujeres que tienen participación en la opinión publicada en los diarios de mayor cobertura nacional en Ecuador? - ¿Cuál es el promedio de edad de la representación femenina en los diarios digitales en el periodo de estudio (2010-2015)? -¿Cuál es el estado civil de las mujeres que aparecen en la opinión publicada de los diarios digitales eluniverso.com y elcomercio.com?

Para el procesamiento de los datos se utilizó el programa estadístico informático SPSS (Statistical Package for the Social Sciences, versión 23). Gracias a este 
software, se realizó el análisis descriptivo a partir de la frecuencia y porcentaje para las variables nominales de interés, así como se extrajo estadísticos de dispersión y tendencia central para la variable numérica.

\subsection{Estado Civil}

La mayoría de las mujeres se declara casada (41,3\%), seguido por las solteras $(38,1 \%)$ y en menor número las divorciadas $(7,9 \%)$ (ver Gráfico 1). Es notablemente alto el porcentaje de casadas, considerando que la literatura (Abramo, 2004; De Anca y Aragón, 2007; Debeljuh, y Jáuregui, 2004; Soler, 2002; Spain y Bianchi, 1996) sostiene que las mujeres tienen mayor dificultad para conciliar el trabajo con la vida matrimonial y la familia. Sin embargo, en el contexto ecuatoriano, la inserción de la mujer en el mundo laboral se ha producido sin que los "roles tradicionalmente imputados a los sexos se presenten inalterados, la mujer como responsable directa o indirecta -a través de otras mujeres- del trabajo doméstico, convive con un imaginario que recoge mucho más la idea de compartir responsabilidades", ya sea con otra persona que trabaja en el servicio doméstico, o algún miembro de la familia (por ejemplo, madre, abuela, tía, prima, cuñada, etc.), inclusive con el cónyuge (Delfino, 2005: 212). Además, algunos expertos manifiestan que un "determinante del éxito profesional femenino es el equilibrio familiar/personal" (Chinchilla et al., 2007). En este sentido, se puede inferir que uno de los factores del éxito profesional [entendido en esta investigación como lo que le otorga al individuo autoridad para hablar sobre un tema específico y en este sentido, para ser una fuente informativa referida en los medios] de la mujer ecuatoriana es tener un buen empleo y una familia consolidada.

El porcentaje de mujeres solteras también es relevante para el análisis, considerando que para Tharenou et al. (1994), las mujeres directivas suelen ser solteras -para ellas tener una familia (por ejemplo, esposo/a; hijo/as) interfiere en su promoción laboral y no le permite "crecer profesionalmente". En cambio, los hombres directivos habitualmente están casados, y no sólo eso, sino que el matrimonio en el caso de los directivos es un factor que influye positivamente en el éxito de su carrera profesional" (Pfeffer y Ross, 1982; Schneer y Reitman, 1993). Así mismo, se señala que, el estado civil de "ser sola" (es decir, soltera y/o no conviviente), permite una mayor disponibilidad para el trabajo, la búsqueda de estabilidad, de ascenso social, de un logro temprano de los proyectos personales, o bien una opción válida para las mujeres que eligieron otorgar al trabajo un papel preponderante en sus estrategias personales (Delfino, 2005).

En cuanto a las mujeres divorciadas (ver Gráfico 1), el porcentaje es mínimo frente a las otras dos categorías en discusión. Esto se contrapone a los académicos que señalan que la mayoría de las veces los altos cargos de liderazgo y dirección los ocupan mujeres divorciadas o separadas (Massolo, 2007; Tharenou et al., 1994; Uriarte y Ruiz, 1999). 
Gráfico 1. Frecuencia del estado civil de las mujeres seleccionadas como fuentes de información por los diarios digitales El Universo y El Comercio (2010 a 2015)

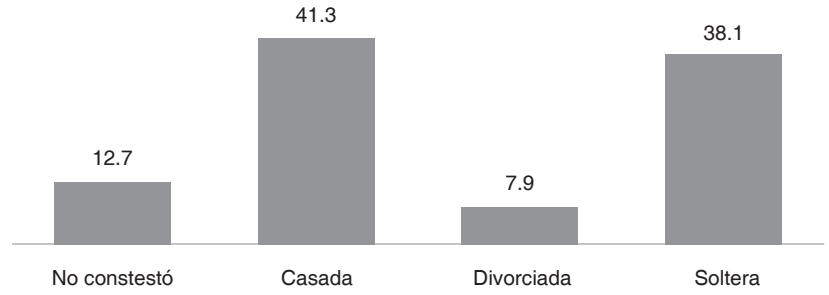

Fuente: Elaboración propia

\subsection{Edad}

El promedio de las mujeres referidas edad (35 a 49 años) de las mujeres ecuapor los medios digitales oscila entre los 40 y 50 años. Esto probablemente se podría asociar de modo positivo con la torianas que aparecen como sujeto de la noticia en los medios (GMMP, 2015). Este rango etario que se considera

Gráfico 2. Promedio de edad de las mujeres seleccionadas como fuentes de información por los diarios digitales El Universo y El Comercio (2010 a 2015)

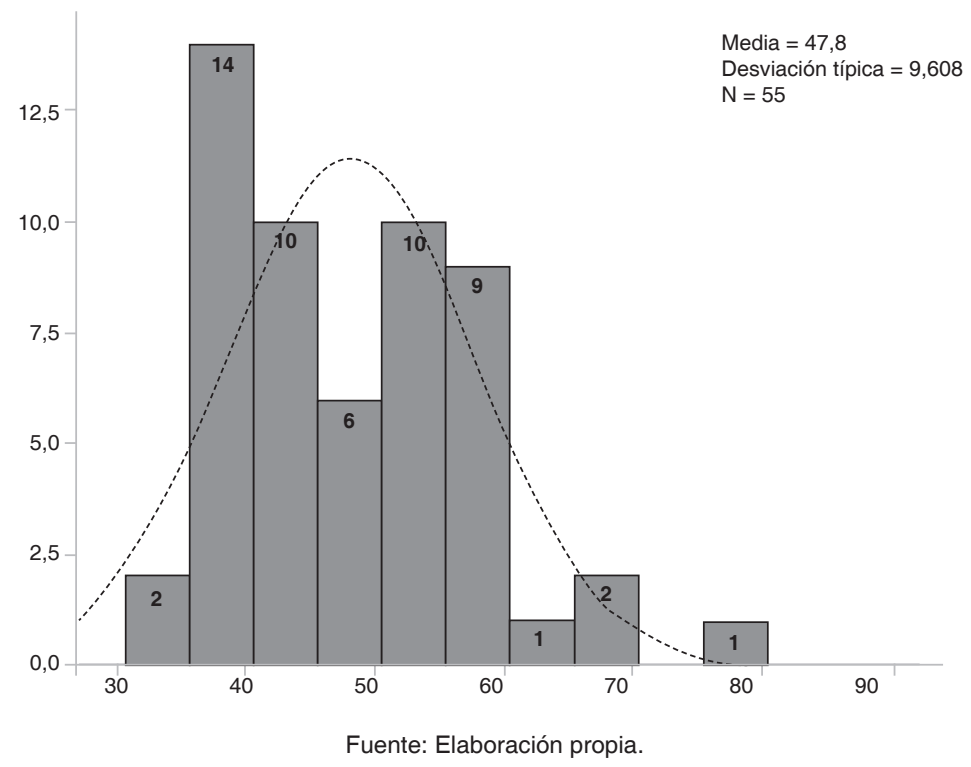


como "edad madura", es un periodo en la vida de la mujer en el que el logro profesional y la satisfacción personal están íntimamente relacionados con su realización como administradoras de su hogar (Delfino, 2005). De hecho, estudios sugieren que el bienestar para es- tas mujeres está centrado en su vida familiar y su estabilidad laboral (Idrovo y Townsend, 2014). De ahí que se pueda estimar que la mujer profesional ecuatoriana habitualmente está directa o indirectamente ligada al trabajo del hogar.

\subsection{Nacionalidad y lugar de nacimiento de las mujeres}

La nacionalidad y lugar de nacimiento de las mujeres tienen una importancia asociada, por sobre todo, a los atajos que provienen del contexto cultural. De hecho, un estudio relativamente reciente analiza la presencia de inmigrantes residentes en cargos de la vida política y pública como una opción cada vez más frecuente de sociedades democráticas (Bird, Saalfeld y Wüst, 2010). De esta muestra, la mayoría de mujeres $(95,2 \%)$ son ecuatorianas, salvo un $4,8 \%$ que po- seen doble ciudadanía o son residentes extranjeras, lo que puede reflejar altos niveles de inclusión formal si se compara a la proporción nacional de estas categorías poblacionales.

En cuanto al lugar de nacimiento, el $47,62 \%$ se ubican en la región Costa, seguido por el $44,44 \%$ en la Sierra. Es mínima $(3,17 \%)$ la presencia de mujeres provenientes del Oriente, incluso menor que las de doble nacionalidad $(4,76 \%)$. No existe una participación-como fuentes

Gráfico 3. Lugar de nacimiento de las mujeres seleccionadas como fuentes de opinión por los diarios digitales El Universo y El Comercio (2010 a 2015)

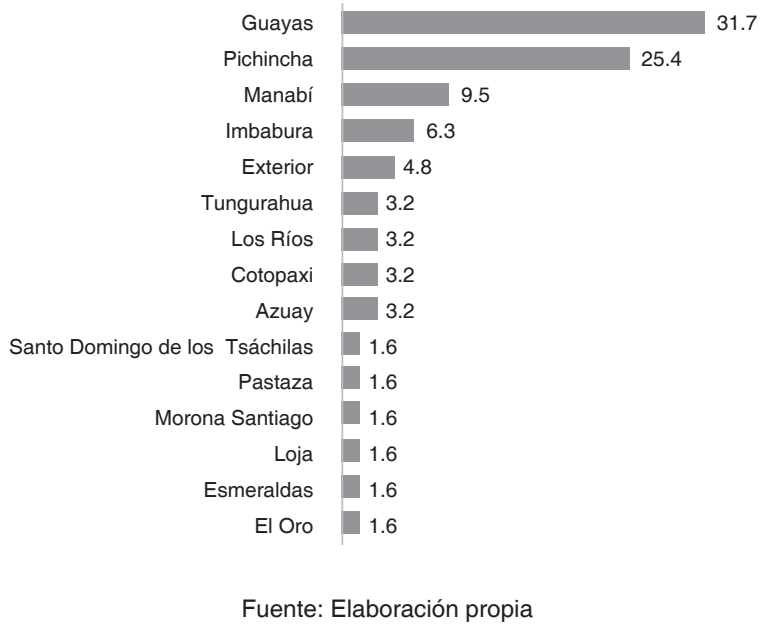


informativas-de mujeres de la región Insular. Esto se puede considerar como producto de "la presencia de fracturas latentes (cleavages) que no han terminado de incorporarse en la comunidad nacional" (Alcántara y Freidenberg, 2001: 27); y en ocasiones también como producto de las configuraciones culturales (por ejemplo, tradiciones, manifestaciones religiosas, etc.) y modelos económicos de la región a la que pertenecen (es decir, costa, sierra, oriente e insular). Sobre todo, entre Quito y Guayaquil, las ciudades más importantes demográficamente, ya que la primera es la capital del país, y la segunda, el principal puerto comercial. De ahí que Pichincha y Guayas -provincias, en las que se ubican Quito y Guayaquil, respectivamente-sobresalgan en las dos primeras posiciones de representación en la muestra (ver Gráfico 3).

\subsection{Auto-identificación étnica}

La participación de mujeres indígenas en el escenario público mediatizado es escasa. Esto es consecuente con el $1,6 \%$ de entidades registrado en la muestra de este estudio (ver Gráfico 4); menos de la mitad de ecuatorianas auto-identificadas indígenas según el Censo Poblacional y de Vivienda (INEC, 2010). Cabe reconocer que las comunidades indígenas se ubicaron en el escenario político y los medios de comunicación en 1990 después del Primer Levantamiento Indígena Nacional (Guerrero, 2000: 99). También hay que señalar que los medios de comunicación se concentran principalmente en zonas urbanas y no rurales que es donde habitualmente se encuentran asentadas las comunidades indígenas.

Por su parte, los resultados de autoidentificación étnica son coincidentes con los datos del Censo de Población y Vivienda (INEC, 2010), donde se estima que la mayor categoría poblacional de los habitantes en Ecuador se autoidentifican mestizos, al igual que en la muestra de este estudio $(96,8 \%)$.

Gráfico 4. Auto-identificación étnica de las mujeres seleccionadas como fuentes de opinión por los diarios digitales

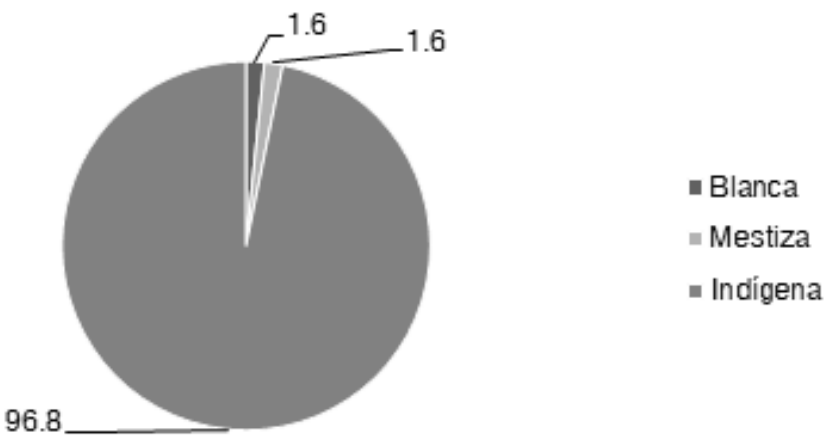

Fuente: Elaboración propia. 


\subsection{Educación}

La educación formal es otro de los indicadores sociodemográficos relacionados con el liderazgo y la representación femenina (Vargas, 2005). En el análisis se pudo observar que la formación profesional de las mujeres referidas por la prensa durante el periodo de estudio
(2010 - 2015) es de tercer nivel o con estudios universitarios de pregrado $(85,71 \%)$, y de cuarto nivel o con especializaciones, maestrías o doctorados $(14,29 \%)$. Se podría decir que uno de los rasgos que los diarios digitales buscan en el perfil de sus informantes mujeres entonces es la preparación académica.

Tabla 2. Profesión de las mujeres seleccionadas como fuentes de opinión por los diarios digitales El Universo y El Comercio (2010 a 2015)

\begin{tabular}{|l|c|}
\hline \multicolumn{1}{|c|}{ Profesión } & $\%$ \\
\hline Abogada & 39,7 \\
\hline Licenciatura en Administración de Empresas & 1,6 \\
\hline Doctora en Medicina & 4,8 \\
\hline Economista & 7,9 \\
\hline Filología Hispánica & 1,6 \\
\hline Ingeniera Civil & 1,6 \\
\hline Ingeniería Comercial & 4,8 \\
\hline Licenciatura en Ciencias de la Educación & 7,9 \\
\hline Licenciatura en Gestión para el Desarrollo Local & 1,6 \\
\hline Licenciatura en Historia y Ciencia Política & 1,6 \\
\hline Maestría en Ciencias Políticas & 1,6 \\
\hline Maestría en Administración Pública & 1,6 \\
\hline Maestría en Estadística y Econometría & 1,6 \\
\hline Maestría en Gestión Administrativa & 1,6 \\
\hline Maestría en Estudios Socio Ambientales & 1,6 \\
\hline Maestría en Derecho & 1,6 \\
\hline Maestría en Derecho de Empresa & 1,6 \\
\hline Maestría en Química & 1,6 \\
\hline Odontóloga & 1,6 \\
\hline Periodista & 9,5 \\
\hline PhD. (c). Geografía Ambiental & 1,6 \\
\hline Psicóloga & 1,6 \\
\hline
\end{tabular}

Fuente: Elaboración propia 
En relación a la formación profesional/ académica, predominan las profesiones de ciencias sociales en relación a las de ciencias naturales. Un informe del Instituto de la Democracia del Consejo Nacional Electoral (IDD-CNE, 2017) sostiene que el " $91 \%$ de los graduados en ingenierías son hombres, aunque del total de la matrícula universitaria más de la mitad corresponde a mujeres" (p. 10). Las mujeres, según el documento del IDD-CNE, se ubican preferentemente en carreras orientadas al bienestar. En este artículo se observa que, si bien no hay un interés relevante por dichas carreras, hay un mayor porcentaje de abogadas
$(39,68 \%)$, y un menor porcentaje de ingenieras, químicas y estadísticas $(7,94 \%)$ (ver Tabla 2). Este resultado coincide con la información estadística que presenta el organismo oficial Sistema Nacional de Información de la Educación Superior del Ecuador (SNIESE) en su Geoportal, que ofrece información estadística de las profesiones segmentadas por sexo (ver Gráfico 5). Así, a partir de esta información, se pudo corroborar que las carreras de Derecho, Periodismo e Información, Economía y Ciencias de la Educación, son las áreas de formación académica/ profesional que las mujeres ecuatorianas más optan.

Gráfico 5. Distribución profesional de acuerdo al sexo

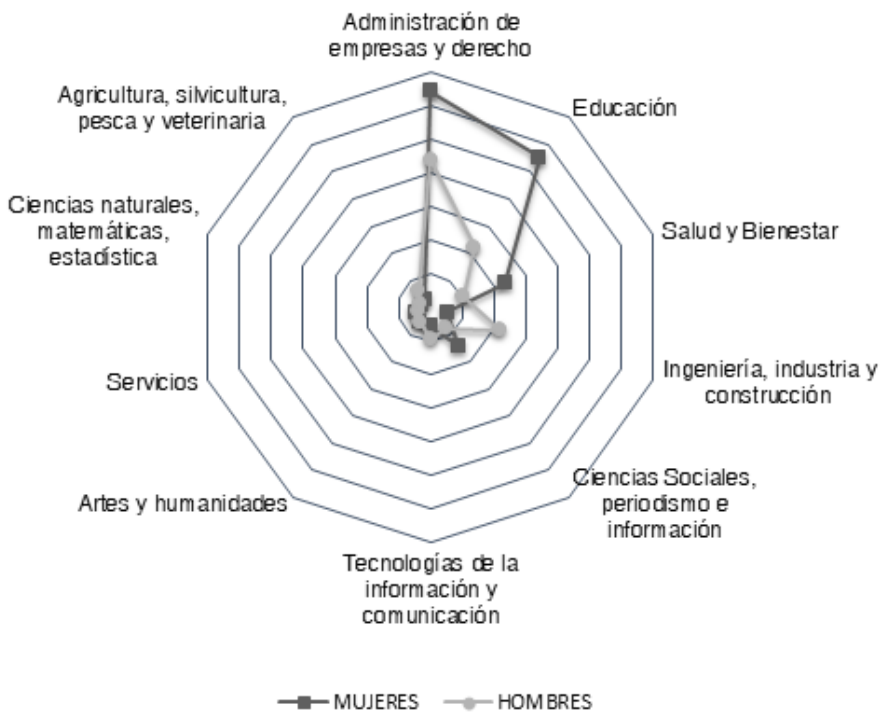

Fuente: Elaborado a partir de los datos del Geoportal del SNIESE.

Este incremento de la preparación profesional de las mujeres, también es visible en la participación que actual- mente tienen en el mercado laboral. De acuerdo con la Encuesta Nacional de Empleo, Desempleo y Subempleo del 
INEC $^{4}$, las mujeres empleadas alcanzaron un $94,6 \%$ hasta septiembre de 2017, frente al 96,8\% de los hombres, solamente dos puntos de diferencia.

\section{Discusión y conclusión}

A lo largo de las dos últimas décadas, la inserción femenina en el ámbito público y político ha tenido un aumento progresivo en Ecuador, al menos en términos de representación descriptiva. Si bien, este crecimiento fue impulsado inicialmente por la creación de la Ley de Amparo Laboral de la Mujer en 1997, la Ley Orgánica de Elecciones en el año 2000 produjo un incremento de la incorporación femenina en los procesos políticos del país, de manera que para el 2007 se logró obtener igualdad de condiciones en cuanto a hombres y mujeres en las listas electorales (Cañete, 2004; De la Cal Pedroso y Sellers, 2011; Htun y Piscopo, 2014; Umpierrez de Reguero, Jara-Alba y Cassis Jurado, 2016).

En esta investigación, se procuró identificar en qué medida los medios toman como referencia a las mujeres en sus publicaciones, y cuál es su perfil sociodemográfico. Así, a partir de un crawling de datos en dos medios de comunicación digital con cobertura nacional -El Universo y El Comercio- se logró identificar que la educación formal y la experiencia que poseen estas muje- res son factores que les permiten ser consideradas como "voces expertas" en el espacio público (similar al argumento de Vargas, 2015). En este sentido, se puede construir hipótesis de testeo en relación al perfil sociodemográfico (es decir, género, auto-identificación étnica, edad, etc.) de estas mujeres como variable(s) independiente(s), y la percepción que tienen los otros sobre ellas como sujetos de una noticia o voces autorizadas para opinar en relación con temas de diversa índole (por ejemplo, salud, economía, familia, etc.) como variable dependiente. También se puede considerar el número de apariciones como variable de respuesta y estimar mediante métodos de estadística inferencial si por ejemplo la edad y experiencia laboral son estadísticamente significativas. De hecho, se puede diseñar experimentos naturales y de campo para mediante el efecto promedio del tratamiento no solo entender sino explicar con más precisión la incidencia de estas variables arriba mencionadas. A nivel metodológico, se puede sugerir trabajar con una muestra censal o al menos probabilística estratificada para precisar aún más los resultados descriptivos encontrados en este trabajo; así recolectar datos de todos los diarios digitales en Ecuador. Con estas tres últimas sugerencias para lineamientos de

4 El diseño para la selección de la muestra de la ENEMDU 2014 es similar al utilizado en el 2013, el método de selección es probabilístico en dos etapas con estratificación geográfica por dominios de estudio y área urbanarural. Los estimadores asociados al diseño se ajustan por una proyección de población calculada según métodos demográficos. Como se puede observar, en general, el nuevo diseño es idéntico al vigente, en ese sentido, los cambios se realizan con el fin de mejorar la precisión de los estimadores y ampliar la medición de los mismos a nivel de zonas de planificación y jurisdicciones de reciente creación. Recuperado el 11 de noviembre de 2017 de http://www.ecuadorencifras.gob.ec//documentos/web-inec/EMPLEO/archivos_ENEMDU/DisenoMuestra.pdf 
futuras investigaciones, se podría pensar en razón de la frecuencia de visibilidad en medios de comunicación digital como proxy de patrones de liderazgo y poder ampliar los recursos de las mismas entidades y sus asesores a la hora de diseñar y ejecutar estrategias para tener un mejor desempeño y rendimiento en la vida política y pública.

En este artículo, se constató que la auto-identificación étnica es un factor que probablemente incide significativamente en la participación de mujeres mediatizada en el espacio público. El porcentaje de mujeres que se auto-identifican indígenas o afro-ecuatorianas y que son referidas por la prensa como fuente de noticia, es mínimo en relación con las que se auto-identifican mestizas, acorde con la información proporcionada por el Censo de Vivienda y Población (INEC, 2010). Esto agrega variables nuevas al análisis tradicional de liderazgo femenino, opinión publicada y medios de comunicación digital. En este caso, los determinantes culturales son otros que por ejemplo en la mayoría de países desarrollados. Ecuador, en este sentido, aparte de ser una nueva democracia, tiene características atractivas como un porcentaje significativo de población indígena con reconocimiento constitucional (Andrade, Grijalva y Storini, 2009). Esto puede funcionar como una prueba de filtro para aquellas teorías existentes que tienen una conceptualización minimalista detrás y que muy probablemente no son tan exigentes para que su adaptación sea más amplia es decir, para que se aplique a un mayor número de casos. De este modo, examinar temas como éste en casos sui generis como Ecuador, puede resultar enriquecedor para la literatura previa por el solo hecho de agregar variables y precisar conjeturas.

Los otros resultados de este artículo, también pueden ser analizados bajo otras ópticas teóricas y metodológicas. El estado civil y la edad de estas 78 mujeres -que son otros de los factores sociodemográficos que caracterizan a la representación de mujeres en los medios de comunicación tradicionales y no tradicionales- pueden resultar interesantes para futuros estudios. Pese a un número creciente de contribuciones que reportan que las mujeres solteras y de menos edad están apareciendo más en los medios digitales (GMMP, 2015), en esta ocasión la mayoría son casadas y pertenecen al rango etario entre los 35 y 50 años. En otras palabras, son personas adultas, que poseen más experiencia que un estudiante universitario por la edad que tienen, y que están ubicadas en este segmento de la población que se reconoce como económicamente activo.

\section{Bibliografía}

Abramo, L. (2004). ¿̇Inserción laboral de las mujeres en América Latina: una fuerza de trabajo secundaria? Estudios Feministas, 12(2), pp. 224-235. https://doi.org/10.1590/ s0104-026x2004000200013

Alcántara Sáez, M. \& Freidenberg, F. (2001). Los partidos políticos en América Latina. América Latina Hoy, (27), pp. 17-35. https://doi.org/10.1590/s0104-62762002000200001 
Andrade, S., Grijalva, A., \& Storini, C. (2009). La nueva Constitución del Ecuador: Estado, derechos e instituciones. Quito: Corporación Editora Nacional.

Barba, J. D., \& Nieto, S. (2006). Mujer, sexualidad, internet y política. Los nuevos electores latinoamericanos. México: Fondo de Cultura Económica.

García-Beaudoux, V. (2017). Ser mujer, dedicarte a la política y no morir en el intento con los medios. Más poder local, (30), pp. 26-27.

Bird, K., Saalfeld, T., \& Wüst, A. M. (Eds.). (2010). The Political Representation of Immigrants and Minorities: Voters, Parties and Parliaments in Liberal Democracies. London: Routledge.

Bystrom, D. G. (2004). Women as Political Communication Sources and Audiences. In Kaid, L. L. (Ed.). Handbook of Political Communication Research. New Jersey-London: Lawrence Erlbaum Associates.

Cañete, María Fernanda (2005). Participación política y ciudadanía. En Prieto, M. (Ed.). Mujeres ecuatorianas: Entre la crisis y las oportunidades 1990-2004. Quito: FLACSO - Sede Ecuador.

Castellanos, A. S. \& C. Ulloa (2016). Representación política de las mujeres a nivel legislativo en Ecuador: un análisis entorno a la promoción de los derechos de la mujer desde 1941 a 2014. Revista de Ciencia Política y Relaciones Internacionales, 5(5), pp. 8-29.

Chinchilla, N., León, C., Torres, E., \& Canela, M. A. (2007). Trayectoria, conciliación y rol directivo. Centro Internacional de Trabajo y Familia IESE, № 47. Pamplona: Universidad de Navarra

De Anca, C., \& Aragón, S. (2007). La mujer directiva en España: catalizadores e inhibidores en las decisiones de trayectoria profesional. Revista Latinoamericana de Administración, (38), pp. 45-64.

De la Cal Pedroso, J., \& Ferrando Sellers, R. (2011). Plan de acción de género en desarrollo de la Cooperación Española en Ecuador 2011-2014. Madrid: Agencia Española de Cooperación Internacional para el Desarrollo.

Debeljuh, P., \& Jáuregui, K. (2004). Trabajo y familia: hacia una cultura familiar amigable en el contexto latinoamericano. Cuadernos de Difusión, 9 (16), pp. 91-91.

Delfino, A. (2005). Mujer y ejecutiva: Trayectoria de género en Brasil. Espacio Abierto, 14 (2).

García-Beaudoux, V. (2017). Ser mujer, dedicarte a la política y no morir en el intento con los medios. Más poder local, (30), pp. 26-27.

GMMP (2015). Proyecto Global de Monitoreo de Medios 2010. Recuperado de: https://www. gammaecuador.org/media/uploads/cyclope_old/adjuntos/GMMP_2010_ecuador.pdf

Guerrero, A. (2000). Los linchamientos en las comunidades indígenas (Ecuador): ¿la política perversa de una modernidad marginal? Bulletin de l'Institut Français d'Études Andines, 29 (3), pp. 463-489. https://doi.org/10.4000/books.ifea.3840

Htun, M., \& Piscopo, J. (2014). Women in politics and policy in Latin America and the Caribbean. In Conflict Prevention and Peace Forum CPPF Working Papers on Women in Politics (Vol. 2).

Instituto de la Democracia del Consejo Nacional Electoral (2017). Datos Electorales. Género y Representación Política. Gaceta OPINIÓN ELECTORAL No. 19. Recuperado de http:// 
ciespal.org/wp-content/uploads/2017/10/Serie-Gaceta-Opini-n-Electoral-No.19Octubre-2017.pdf

Leopold, T. A., Ratcheva, V., \& Zahidi, S. (2016). The Global Gender Gap Report 2016. In World Economic Forum.

Llanos, B. (2014). Ojos que (aún) no ven. Nuevo reporte de 8 países: género, campañas electorales y medios en América Latina. La representación imperfecta. Logros y desafíos de las mujeres políticas, pp. 81-104.

Massolo, A. (1998). Pluralidad política y pluralidad de género a favor de ayuntamientos democráticos. Dalia Barrera Bassols y Alejandra Massolo (coords), Mujeres que gobiernan municipios. Experiencias, aportes y retos, PIEM, El Colegio de México, México, DF.

Niemi, M. K., \& Pitkänen, V. (2017). Gendered use of experts in the media: Analysis of the gender gap in Finnish news journalism. Public Understanding of Science, 26(3), pp. 355-368. https://doi.org/10.1177/0963662515621470

Pfeffer, J., \& Ross, J. (1982). The effects of marriage and a working wife on occupational and wage attainment. Administrative Science Quarterly, 27(1), pp. 66 - 80. https://doi. org/10.2307/2392546

Ross, K., \& Carter, C. (2011). Women and news: A long and winding road. Media, Culture छ Society, 33(8), pp. 1148-1165. https://doi.org/10.1177/0163443711418272

Schneer, J. A., \& Reitman, F. (1993). Effects of alternate family structures on managerial career paths. Academy of Management Journal, 36(4), pp. 830-843. https://doi. org/10.2307/256760

Spain, D., \& Bianchi, S. (1996). Balancing Act: Motherhood, Marriage, and Employment among American Women. New York: Russell Sage Foundation.

Tharenou, P., Latimer, S., \& Conroy, D. (1994). How do you make it to the top? An examination of influences on women's and men's managerial advancement. Academy of Management Journal, 37(4), pp. 899-931. https://doi.org/10.2307/256604

Tribunal Contencioso Electoral del Ecuador. Codificación Ley Orgánica de Elecciones. Registro Oficial Año I N 117, Quito, Ecuador, Martes 11 de julio del 2000. Recuperado de http://www.tce.gob.ec/jml/bajar/transparencia/LEY-DE-ELECCIONES.pdf

Umpierrez de Reguero, S., Jara-Alba, C., \& Cassis Jurado, A. (2016). Ecuador, mujeres y representación legislativa (1979-2015). Revista Enfoques: Ciencia Política y Administración Pública, 14(24), pp. 13-40.

Uriarte, E., \& Ruiz, C. (1999). Mujeres y hombres en las élites políticas españolas: ¿Diferencias o similitudes? REIS: Revista Española de Investigaciones Sociológicas, (88), pp. 207-232. https://doi.org/10.2307/40184208

Vargas Muñoz, A. (2015). Representaciones mediáticas de la participación política de la mujer. Revista Gestión de las Personas y Tecnología, 8 (22), p. 13.

World Association for Christian Communication (2015). The Global Media Monitoring Project. https://doi.org/10.1163/2211-2685_eco_w.27

Zinko, R., Ferris, G. R., Blass, F. R., \& Dana Laird, M. (2007). Toward a theory of reputation in organizations. Human Resources Management, (26), pp. 163-204. 


\section{Anexo 1}

Muestra del estudio

\begin{tabular}{|c|c|c|c|}
\hline \multirow{2}{*}{$\begin{array}{l}\text { Ranking entre las } \\
444 \text { entidades }\end{array}$} & \multirow{2}{*}{$\begin{array}{l}\text { Nombre de la entidad } \\
\text { identificada }\end{array}$} & \multicolumn{2}{|c|}{ Frecuencia de aparición } \\
\hline & & $\mathbf{N}$ & $\%$ \\
\hline 16 & Lourdes Tibán & 986 & 5,0 \\
\hline 23 & Nathalie Cely & 824 & 4,1 \\
\hline 27 & Gabriela Rivadeneira & 760 & 3,8 \\
\hline 35 & María Paula Romo & 692 & 3,5 \\
\hline 44 & Marcela Aguiñaga & 582 & 2,9 \\
\hline 58 & María Fernanda Espinosa & 521 & 2,6 \\
\hline 69 & Betty Carrillo & 473 & 2,4 \\
\hline 73 & Viviana Bonilla & 468 & 2,4 \\
\hline 77 & Cynthia Viteri & 451 & 2,3 \\
\hline 78 & Rossana Alvarado & 450 & 2,3 \\
\hline 81 & Silvia Salgado & 430 & 2,2 \\
\hline 94 & Gina Godoy & 390 & 2,0 \\
\hline 99 & Tania Arias & 378 & 1,9 \\
\hline 107 & Marcela Miranda & 354 & 1,8 \\
\hline 110 & Paola Pabón & 347 & 1,7 \\
\hline 121 & Ximena Ponce & 322 & 1,6 \\
\hline 124 & Mónica Franco & 318 & 1,6 \\
\hline 127 & Betty Amores & 315 & 1,6 \\
\hline 131 & Irina Cabezas & 303 & 1,5 \\
\hline 134 & Natalia Emme & 301 & 1,5 \\
\hline 136 & Betty Tola & 301 & 1,5 \\
\hline 142 & María Augusta Calle & 287 & 1,4 \\
\hline 156 & Carina Vance & 269 & 1,4 \\
\hline 158 & Andrea Rivera & 263 & 1,3 \\
\hline 159 & María Corina Machado & 262 & 1,3 \\
\hline 161 & Gloria Vidal & 257 & 1,3 \\
\hline 167 & Martha Roldós & 250 & 1,3 \\
\hline 175 & Mery Zamora & 238 & 1,2 \\
\hline 180 & Roxana Silva & 232 & 1,2 \\
\hline 182 & Scheznarda Fernández & 231 & 1,2 \\
\hline 183 & Mónica Banegas & 230 & 1,2 \\
\hline 189 & Mónica Encalada & 225 & 1,1 \\
\hline 194 & Lucy Blacio & 218 & 1,1 \\
\hline 196 & Alexandra Ocles & 215 & 1,1 \\
\hline 197 & Paola Carvajal & 214 & 1,1 \\
\hline 202 & Gloria Sabando & 207 & 1,0 \\
\hline 203 & María Leonor Jiménez & 206 & 1,0 \\
\hline 216 & Marcia Caicedo & 200 & 1,0 \\
\hline
\end{tabular}




\begin{tabular}{|c|c|c|c|}
\hline 225 & Marisol Peñafiel & 190 & 1,0 \\
\hline 226 & Nubia Villacís & 190 & 1,0 \\
\hline 230 & Aminta Buenaño & 188 & 0,9 \\
\hline 237 & Nívea Vélez & 183 & 0,9 \\
\hline 238 & María Emma Mejía & 182 & 0,9 \\
\hline 246 & Rocío Valarezo & 177 & 0,9 \\
\hline 248 & Katiuska King & 176 & 0,9 \\
\hline 251 & María Elsa Viteri & 175 & 0,9 \\
\hline 259 & Delcy Rodríguez & 169 & 0,9 \\
\hline 260 & Lorena Tapia & 168 & 0,8 \\
\hline 266 & Patricia Parrales & 166 & 0,8 \\
\hline 267 & Caroline Chang & 165 & 0,8 \\
\hline 270 & Cristina Reyes & 163 & 0,8 \\
\hline 271 & Tatiana Ordeñana & 162 & 0,8 \\
\hline 274 & \begin{tabular}{|l|} 
Ivonne Baki \\
\end{tabular} & 161 & 0,8 \\
\hline 275 & Nina Pacari & 161 & 0,8 \\
\hline 277 & Soledad Buendía & 161 & 0,8 \\
\hline 285 & Mónica Chuji & 156 & 0,8 \\
\hline 293 & Verónica Sión & 150 & 0,8 \\
\hline 297 & Gladys Terán & 149 & 0,8 \\
\hline 315 & Mae Montaño & 144 & 0,7 \\
\hline 318 & Ruth Hidalgo & 142 & 0,7 \\
\hline 322 & Alexandra Arce & 140 & 0,7 \\
\hline 327 & Daniela Chacón & 139 & 0,7 \\
\hline 343 & María Sol Corral & 133 & 0,7 \\
\hline 345 & Gabriela Pazmiño & 133 & 0,7 \\
\hline 353 & Mary Verduga & 130 & 0,7 \\
\hline 355 & Diana Atamaint & 130 & 0,7 \\
\hline 356 & María José Carrión & 130 & 0,7 \\
\hline 362 & Pamela Falconí & 128 & 0,6 \\
\hline 364 & Lorena Escudero & 127 & 0,6 \\
\hline 376 & Johana Pesántez & 123 & 0,6 \\
\hline 384 & Mónica Vargas & 121 & 0,6 \\
\hline 394 & Gloria Gallardo & 118 & 0,6 \\
\hline 407 & Martha Bucaram & 114 & 0,6 \\
\hline 408 & María Alejandra Vicuña & 114 & 0,6 \\
\hline 425 & María Isabel Salvador & 111 & 0,6 \\
\hline 434 & Susana Dueñas & 108 & 0,5 \\
\hline 443 & Jeannette Sánchez & 105 & 0,5 \\
\hline 444 & Ruth Seni & 105 & 0,5 \\
\hline \multicolumn{2}{|l|}{ Total } & 19857 & 100,0 \\
\hline
\end{tabular}

